\title{
Multimode quantum state tomography using unbalanced array detection
}

\author{
Brandon S. Harms, Blake E. Anthony, Noah T. Holte, Hunter A. Dassonville, and Andrew M. C. Dawes* \\ Pacific University, Department of Physics, Forest Grove, Oregon, 97116, USA
}

(Received 25 July 2014; published 10 November 2014)

\begin{abstract}
We measure the joint $Q$ function of a multispatial-mode field using a charge-coupled device array detector in an unbalanced heterodyne configuration. The intensity pattern formed by interference between a weak signal field and a strong local oscillator is resolved using Fourier analysis and used to reconstruct quadrature amplitude statistics for 22 spatial modes simultaneously. The local oscillator and signal propagate at an angle of $12 \mathrm{mrad}$, thus shifting the classical noise to modes that do not overlap with the signal. In this configuration, balanced detection is not necessary.
\end{abstract}

DOI: 10.1103/PhysRevA.90.053818

PACS number(s): 42.50.Ar, 03.65.Wj, 03.65.Ta

Multimode quantum optical systems are increasingly important in applications such as slow and stored light devices [1]. A complete understanding of these devices requires a fidelity measurement of the stored quantum state, but there has not yet been a simple way to measure the quantum state of a multispatial mode field. Balanced homodyne detection (BHD) is the standard measurement technique used to construct the complete quantum-mechanical state of light [2]. This technique has one primary weakness: multimode fields or fields in an unknown spatial mode suffer significant losses due to mode mismatch between the local oscillator (LO) and the signal field [2,3].

Balanced array detection overcomes mode-matching losses and has been used to perform measurements of more than two modes at once [4]. It is possible to obtain simultaneous (but not joint) measurements of the Wigner functions of many modes by using array detectors [5,6]. It has also been shown theoretically that array detectors may be used to measure the joint $Q$ function of a multimode field [7]. One of the difficulties of these methods is the need to balance two array detectors and perform pixel-by-pixel subtraction to eliminate the classical intensity noise of the LO [8]. These alignment requirements become prohibitive when trying to measure two transverse directions. This difficulty was removed by Beck et al. who achieved the same effect with a single array, using unbalanced detection, by arranging the LO and signal fields to isolate the classical LO noise [9].

In this article, we describe a multispatial-mode detection method that is analogous to the multitemporal-mode detection method of Beck et al. We simultaneously measure many spatial modes without having to vary the propagation direction or the phase of the LO; this reduces the amount of data that need to be acquired and enables simultaneous joint measurements of two modes. This measurement technique can measure joint statistics between two independent spatial modes. Knowledge of these intermode correlations could provide a new route to information storage in a wide variety of systems.

\footnotetext{
*dawes@pacificu.edu; www.amcdawes.com

Published by the American Physical Society under the terms of the Creative Commons Attribution 3.0 License. Further distribution of this work must maintain attribution to the author(s) and the published article's title, journal citation, and DOI.
}

To develop the theory of unbalanced array detection, we consider the spatial intensity $S(\mathbf{x})$ incident on the array detector due to the combination of an LO field $E_{\mathrm{LO}}$ and the signal field $E_{S}$. For simplicity, we describe only one transverse coordinate (i.e., horizontal); the generalization to both transverse coordinates is straightforward. If the LO is normally incident on the detector and the signal field propagates at a small angle to the LO, the spatial intensity at the detector is given by

$$
\begin{aligned}
S(\mathbf{x})= & \left|E_{\mathrm{LO}}(\mathbf{x})+E_{S}(\mathbf{x}) \exp \left(i \mathbf{k}_{\mathbf{S}} \cdot \mathbf{x}\right)\right|^{2} \\
= & \left|E_{\mathrm{LO}}(\mathbf{x})\right|^{2}+\left|E_{S}(\mathbf{x})\right|^{2} \\
& +\left[E_{\mathrm{LO}}^{*}(\mathbf{x}) E_{S}(\mathbf{x}) \exp \left(i \mathbf{k}_{\mathbf{S}} \cdot \mathbf{x}\right)+\text { c.c. }\right] .
\end{aligned}
$$

The Fourier transform of this measurement yields

$$
\begin{aligned}
\widetilde{S}(\mathbf{k})= & \widetilde{E}_{\mathrm{LO}}^{*}(-\mathbf{k}) \otimes \widetilde{E}_{\mathrm{LO}}(\mathbf{k})+\widetilde{E}_{S}^{*}(-\mathbf{k}) \otimes \widetilde{E}_{S}(\mathbf{k}) \\
& +f\left(\mathbf{k}-\mathbf{k}_{\mathbf{S}}\right)+f^{*}\left(-\mathbf{k}-\mathbf{k}_{\mathbf{S}}\right),
\end{aligned}
$$

where $f(\mathbf{k})=\widetilde{E}_{\mathrm{LO}}^{*}(-\mathbf{k}) \otimes \widetilde{E}_{S}(\mathbf{k})$ and $\otimes$ denotes the convolution. The first of the three terms peaks at $\mathbf{k}=\mathbf{0}$ and contains the second-order classical LO noise that would be removed in balanced detection. The second term is negligible if the signal field is weak. If $\mathbf{k}_{\mathbf{S}}$ is large enough, the function $f$ contains all information on the heterodyned signal. This function has a peak value at $\mathbf{k}=\mathbf{k}_{\mathbf{S}}$ and is therefore separate from the classical noise at $\mathbf{k}=0$. Just as Beck et al. [9] eliminated classical LO noise by temporally separating the signal and LO fields, we eliminate classical LO noise by separating the LO and signal fields by their propagation direction: They have different transverse components of their propagation wave vector [10].

To describe the detection process from the perspective of quantum mechanics, we consider the spatial pattern detected at an array, as in Fig. 1, where $\hat{n}_{j}=\hat{a}_{j}^{\dagger} \hat{a}_{j}$ is the operator corresponding to the number of photons incident on pixel $j$ of the array. Each pixel measures a different spatial position $x_{j}=$ $j \delta x$ and there are $N$ pixels labeled by $-N / 2 \leqslant j<N / 2$. We can also express the measured field in terms of plane-wave modes $\hat{b}_{k},-N / 2 \leqslant k<N / 2$ using the Fourier relation

$$
\hat{a}_{j}=\sum_{k} \exp [-i 2 \pi j k / N] \hat{b}_{k} \text {. }
$$

We assume the LO occupies the $2 M+1$ plane-wave modes near the center of the spectral window. The signal occupies the 


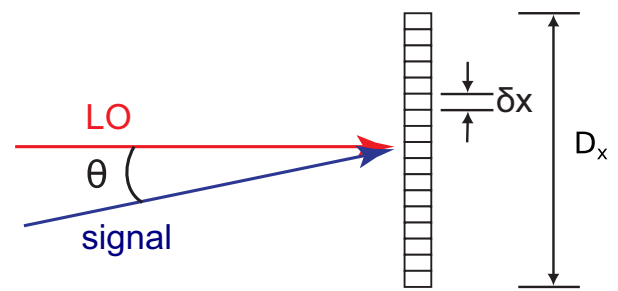

FIG. 1. (Color online) A normally incident plane-wave local oscillator (LO) interferes with a signal field at angle $\theta$. The array detector pixels measure $\delta x$ and the detector size is $D_{x}$.

plane-wave modes with positive $k$ and the plane-wave modes with negative $k$ contain only the vacuum field. This is explicitly written as

$$
\hat{b}_{k}= \begin{cases}\hat{b}_{k}^{(\mathrm{vac})} & -N / 2 \leqslant k<-M, \\ \hat{b}_{k}^{(\mathrm{LO})} & -M \leqslant k \leqslant M, \\ \hat{b}_{k}^{(s)} & M<k<N / 2 .\end{cases}
$$

The operator we measure corresponds to the inverse Fourier transform of $\hat{n}_{j}$

$$
\hat{K}_{p}=\frac{1}{\sqrt{N}} \sum_{j} \exp [i 2 \pi p j / N] \hat{n}_{j} .
$$

Combining Eqs. (3) and (4) yields an expression for the per-pixel number operator $\hat{n}_{j}$ in terms of plane-wave mode operators $\hat{b}_{k}$. The terms that combine two weak fields $\hat{b}_{k}^{(\mathrm{vac})}$ and $\hat{b}_{k}^{(s)}$ are discarded as second order. From here, Eq. (5) is used to relate $\hat{K}_{p}$ in terms of the $\hat{b}_{k}$ 's. Finally, the large-amplitude of the LO field means the LO operators $\hat{b}_{k}^{(\mathrm{LO})}$ can be replaced with their coherent state amplitudes $\beta_{k}$. For $p>2 M$ we find

$$
\hat{K}_{p}=\sum_{k=-M}^{M}\left(\beta_{k}^{*} \hat{b}_{k+p}^{(s)}+\beta_{k} \hat{b}_{k-p}^{\dagger(\mathrm{vac})}\right) .
$$

If the LO occupies only a single $(k=0)$ plane-wave mode, then Eq. (6) simplifies to

$$
\hat{K}_{p}=\beta_{0}^{*} \hat{b}_{p}^{(s)}+\beta_{0} \hat{b}_{-p}^{\dagger(\mathrm{vac})} .
$$

For an LO in a single plane-wave mode, a measurement of $\hat{K}_{p}$ (the Fourier transform of the photocount data) returns a complex number. Using Eq. (7), this complex number can be interpreted as a measurement of $\hat{b}_{p}^{(s)}$, the signal-mode annihilation operator, plus a vacuum contribution [the second term in Eq. (7)]. The annihilation operator is itself the sum of the two field quadrature amplitudes

$$
\hat{b}_{p}^{(s)}=\frac{1}{\sqrt{2}}\left(\hat{x}_{p}+i \hat{y}_{p}\right) .
$$

Therefore, the real and imaginary components of each $\hat{K}_{p}$ correspond to the simultaneous measurement of the quadrature amplitudes $x_{p}$ and $y_{p}$. Of course, these observables are noncommuting so the ability to measure them simultaneously comes at the price of additional vacuum noise [2,11]. It is precisely this additional noise that prevents reconstruction of the Wigner function, though reconstruction of the $Q$ function is still possible.

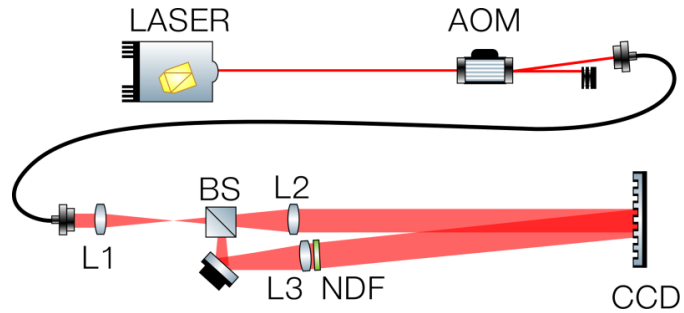

FIG. 2. (Color online) Experimental setup: A 780-nm external cavity diode laser is coupled into single-mode fiber, gated using an 80-MHz acoustooptic modulator (AOM), and split into LO and signal fields that interfere on a CCD array. Lens focal lengths are L1 50, L2 250, and L3 $300 \mathrm{~mm}$.

It is important to note that each Fourier-transformed exposure of the array returns a set of $(N / 2)-M$ complex numbers. Each of these, indexed by $p$, has a real and imaginary part that corresponds to the signal-mode field quadrature $\left(x_{p}, y_{p}\right)$. If one value of $p$ is selected, and the corresponding $\left(x_{p}, y_{p}\right)$ pairs are histogrammed, the result tends toward the $Q$ distribution for the field quadratures of mode $p$. Because the data for all values of $p$ are collected simultaneously, any mode $p$ can be analyzed from a single set of data. Additionally, joint $Q$ distributions can be computed for any pair or combination of modes.

Our implementation of this system, shown in Fig. 2, begins with a 780-nm external-cavity diode laser. The laser output is sent through an acoustooptic modulator $(80 \mathrm{MHz})$. The first-order diffracted beam is then spatially filtered using an optical fiber (780-HP). After the fiber output, a telescope, starting with a 50-mm focal length lens, expands the beam diameter to $1 \mathrm{~cm}$. A 50/50 beam splitter separates the LO from the signal and a $250-\mathrm{mm}$ focal length lens collimates the LO beam after expansion. The signal beam remains slightly diverging after passing through a $300-\mathrm{mm}$ focal length lens. The signal, as a diverging Gaussian beam, occupies a range of plane-wave modes and so provides a simple multimode signal for our demonstration of this method. This approach also maintains a stable phase relationship between the LO and all signal modes. The signal is attenuated by a factor of $10^{5}$ using neutral density filters. The signal and LO interfere on a charge-coupled device (CCD) camera [12]. The CCD is a $1340 \times 400$ array of $\delta x=20 \mu \mathrm{m}$ square pixels with quantum efficiency of $98 \%$ at $780 \mathrm{~nm}$, cooled to $-120^{\circ} \mathrm{C}$ to achieve a dark current rate of two to three electrons per pixel per hour.

The LO power incident on the CCD is $\sim 0.5 \mu \mathrm{W}$ and the LO and signal beams interfere on the CCD at an angle of $\sim 13 \mathrm{mrad}$. The AOM signal is modulated by a function generator (Rigol DG4062) to create a $7.5 \mathrm{~ms}$ rectangular pulse. The pulse is triggered $5 \mathrm{~ms}$ after the start of the $20 \mathrm{~ms}$ exposure to keep the CCD dark during readout.

The largest noise source in our measurements is the electronic readout noise. We characterize this noise by measuring the variance in photocounts $\Delta n_{j}$ with the LO illuminating the CCD and without illumination. The variance with illumination is an average of $15 \mathrm{~dB}$ above the variance without illumination so our signal-to-noise ratio is more than adequate. 


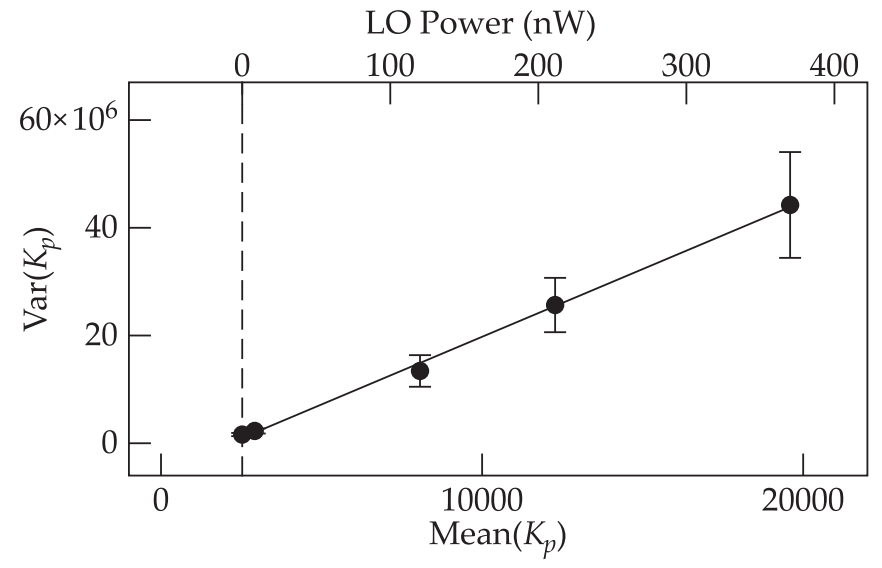

FIG. 3. Linear relationship between the variance of $K_{p}$ versus the mean of $K_{p}$ demonstrates shot-noise-limited detection for modes above $p=200$. The upper horizontal axis indicates LO power for comparison and the gray dashed line indicates zero LO power (i.e., the electronic noise floor). Data shown are for $p=210$, the same mode selected for analysis in Fig. 4.

If the signal is blocked, the signal mode entering the detector is then the vacuum mode, and the quadrature amplitudes measured should be zero. Despite our spatial filtering, the residual spatial components in the LO beam leave a background signal in the FFT output that should be subtracted before computing the $Q$ function. To carry out this correction, we collect 500 exposures with the signal blocked (i.e., the signal in the vacuum state) and average the FFT output across these 500 exposures. This vacuum average $\left\langle\hat{K}_{p}\right\rangle_{\text {vac }}$ is then subtracted from subsequent data prior to further calculations.

We collect 1000 to 8000 shots of CCD data from an ROI at the center of the CCD (600 pixels wide by 10 pixels tall), sum vertically the ten rows to obtain $N=600$ data points, and compute the FFT of the resulting 600-element array. The output of this calculation is an array of 600 complex values of $K_{p}$ where the first 300 values correspond to unique modes indexed by $p$ [13].

Classical noise in the LO is canceled for modes where $p>$ $M$, we find these are modes above $p=200$. We demonstrate that such modes are shot-noise-limited by plotting the variance of the magnitude $\left|K_{p}\right|$ versus the mean of magnitude $\left|K_{p}\right|$ [14]. This is analogous to performing a narrow-band measurement of the noise power versus optical power. It is also important to note that $\left|K_{p}\right|$ itself is not the measured photon number and therefore the variance and mean of $\left|K_{p}\right|$ are not expected to be equal. We place the signal modes in a region of the mode spectrum (the $K_{p}$ 's) that is flat and therefore free of classical noise on the LO, $p>2 M$ as for Eq. (6). Figure 3 shows the linear relationship between these quantities. Furthermore, the electronic noise floor is illustrated by the nonzero variance even when the LO is blocked (zero LO power).

We compute the quadratures by scaling the data by the total number of photons $n_{t}$ detected per shot in the CCD region of interest. For weak signal fields, $n_{t}$ is essentially equal to the average number of photons in the $\mathrm{LO}$ and therefore related to the classical LO amplitude as

$$
|\beta|=\sqrt{n_{t}} .
$$
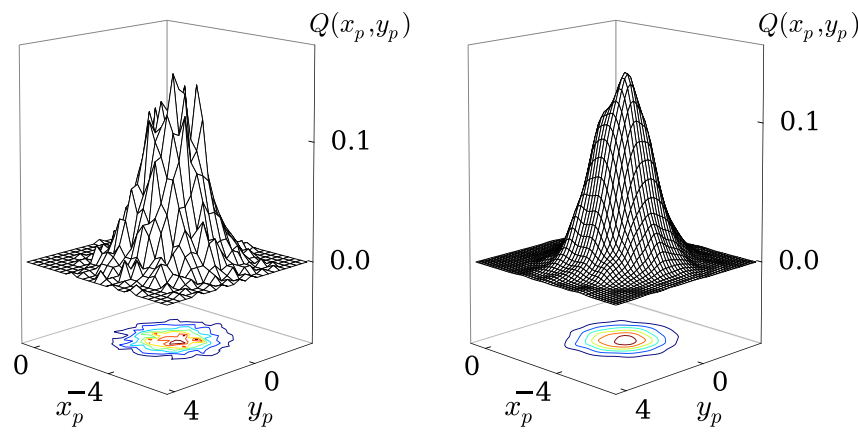

FIG. 4. (Color online) Figure of single-mode $Q$ function. The mode selected here has an average of $\langle n\rangle=7.2$ photons and $\Delta n=$ 2.8 , within $4 \%$ of the quantum noise limit. Shown on the left is the raw-data histogram and on the right is a kernel density estimate calculated from the raw data.

Therefore, the quadratures in Eq. (8) are calculated from experimental values of $K_{p}$ as

$$
\begin{aligned}
& x_{p}=\sqrt{\frac{2}{n_{t}}} \operatorname{Re}\left(K_{p}-\left\langle K_{p}\right\rangle_{\mathrm{vac}}\right), \\
& y_{p}=\sqrt{\frac{2}{n_{t}}} \operatorname{Im}\left(K_{p}-\left\langle K_{p}\right\rangle_{\mathrm{vac}}\right) .
\end{aligned}
$$

From the quadratures, we compute the $Q$ function $Q\left(x_{p}, y_{p}\right)$ for a specific mode $p$. This calculation is done in two ways. First, we simply histogram the $x_{p}$ and $y_{p}$ pairs using 30 bins in each dimension. For large data sets, this histogram will approximate $Q\left(x_{p}, y_{p}\right)$. Additionally, we calculate $Q\left(x_{p}, y_{p}\right)$ by applying a kernel density estimate (KDE) to the $x_{p}$ and $y_{p}$ pairs. This method essentially places a narrow Gaussian function at each point $\left(x_{p}, y_{p}\right)$ and computes the sum. The width of the Gaussian kernel is determined using Scott's rule and results in some smoothing relative to our raw histogram [15].

In Figs. 4, 6, and 7, both the raw-data histogram and the KDE versions of the $Q$ function are presented for comparison. Shown in Fig. 4 is $Q\left(x_{p}, y_{p}\right)$ for a mode with $\langle n\rangle=7.2$ photons and $\Delta n=2.8$, within $4 \%$ of the quantum noise limit. The $Q$ function can be computed for any mode $p$ using the same set of

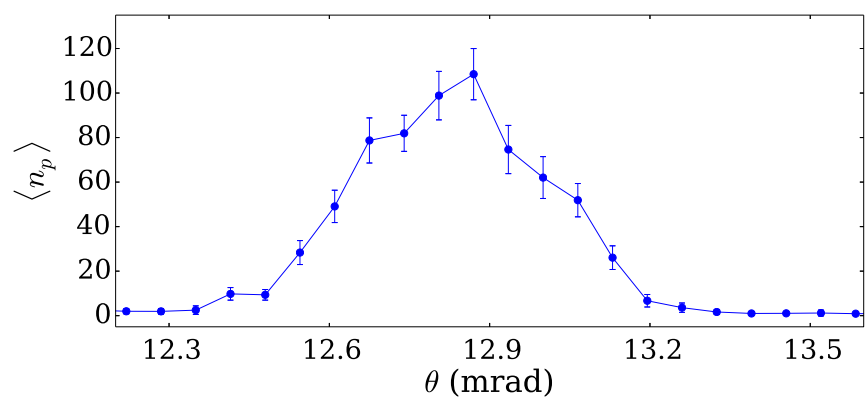

FIG. 5. (Color online) Average photon number vs. plane-wave mode angle. For the 15 modes occupied by the signal field we plot $\left\langle n_{p}\right\rangle$ with error bars given by $\Delta n_{p}$. It should be noted that the observed $\Delta n_{p}$ values vary with $\left\langle n_{p}\right\rangle$ in accordance with the quantum noise limit. 

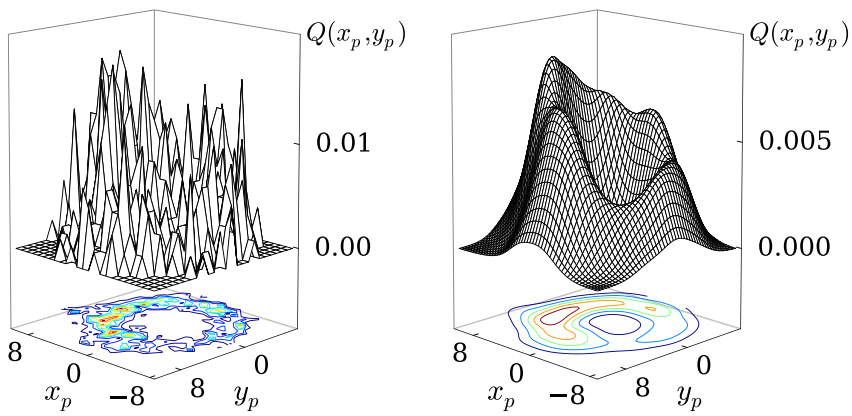

FIG. 6. (Color online) $Q$ function for mode with randomized phase. With randomized phase, the $Q$ function takes on a donut shape centered at the origin. The consistent radius indicates a stable photon number while the angular spread indicates the changing phase.

data. Because data are collected for all modes simultaneously, we can also compute a quantity of interest (such as average photon number $\left\langle n_{p}\right\rangle$ ) and measure that quantity for all modes simultaneously.

The mode resolution is determined by the Fourier sampling frequency at the detector. With $N=600$ detected pixels, each $\delta x=20$ microns wide, the reconstructed modes are plane waves that propagate at an angle of $\theta_{p}=p \lambda /(N \delta x)$ relative to the LO. In Fig. 5 we plot the average photon number $\left\langle n_{p}\right\rangle$ versus plane-wave mode angle $\theta_{p}$. This illustrates the mode spectrum of the signal field. In particular, with a multimode field there are several modes with comparable amplitudes. While Fig. 5 shows the 22 modes near the signal, we measure 300 modes simultaneously; highly multimode signals can be measured using this technique.

To demonstrate the detection of a signal with variable phase, we dither the position of a piezomounted mirror in the signal beam path and observe modulation through $2 \pi$ rad of phase. The $Q$ function calculated from such data is shown in Fig. 6. The characteristic donut shape corresponds to phase noise, although the phase modulation is not completely random as evidenced by the peaks present in the $Q$ function.
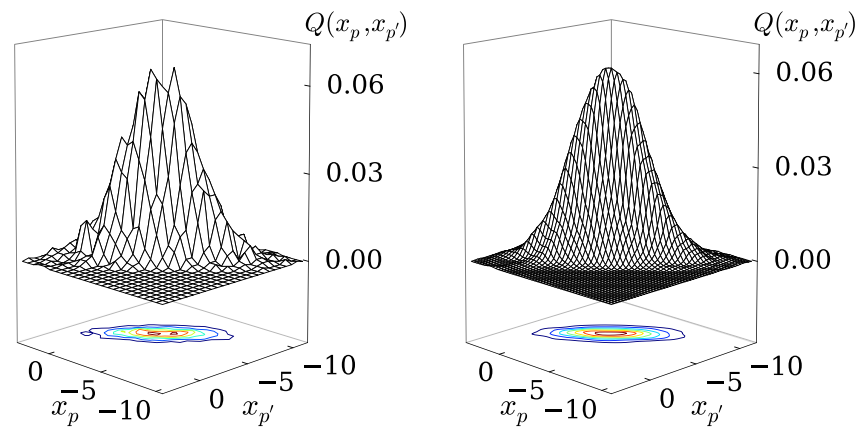

FIG. 7. (Color online) Two-mode joint $Q$ function. For adjacent modes $\left(p=197\right.$ and $\left.p^{\prime}=198\right)$ the joint $Q$ function illustrates the correlation between the real parts, $x_{p}$, of each mode.

It is important to note that we can also compute any quantity that is a function of the quadratures $x_{p}$ and $y_{p}$. One such quantity is the joint $Q$ function, $Q\left(x_{p}, x_{p^{\prime}}\right)$. The joint $Q$ function shown in Fig. 7 is for two nearby modes $p$ and $p^{\prime}=p+1$. As expected, these modes exhibit strong positive correlation between $x_{p}$ and $x_{p^{\prime}}$.

In conclusion, we point out that this method could be used to measure the quantum state of light stored in a multimode quantum memory such as that described in [1]. The peak sensitivity of our CCD system is near the 780 and $795 \mathrm{~nm}$ resonances of $\mathrm{Rb}$. Very recently, quantum-state tomography measurements have been performed on single-photon states retrieved from a stored-light system [16]. Multimode measurements of such systems could be made using methods presented in this article and would yield new information about correlations between modes retrieved from stored-light systems.

\section{ACKNOWLEDGMENTS}

We thank M. Beck for helpful discussions. This material is based upon work supported by the National Science Foundation under Grant No. 1205828. Additional financial support was provided by the Research Corporation for Science Advancement, the Pacific Research Institute for Science and Mathematics, and the M.J. Murdock Charitable Trust.
[1] A. Grodecka-Grad, E. Zeuthen, and A. S. Sørensen, Phys. Rev. Lett. 109, 133601 (2012).

[2] U. Leonhardt, Measuring the Quantum State of Light, Vol. 22, (Cambridge University Press, Cambridge, England, 1997).

[3] M. Beck, Phys. Rev. Lett. 84, 5748 (2000).

[4] M. G. Raymer, J. Cooper, and M. Beck, Phys. Rev. A 48, 4617 (1993).

[5] A. M. Dawes and M. Beck, Phys. Rev. A 63, 040101 (2001).

[6] A. M. Dawes, M. Beck, and K. Banaszek, Phys. Rev. A 67, 032102 (2003).

[7] C. Iaconis, E. Mukamel, and I. A. Walmsley, J. Opt. B: Quantum Semiclass. Opt. 2, 510 (2000).

[8] H. P. Yuen and V. W. S. Chan, Opt. Lett. 8, 177 (1983).

[9] M. Beck, C. Dorrer, and I. A. Walmsley, Phys. Rev. Lett. 87, 253601 (2001).
[10] A. M. C. Dawes, N. T. Holte, and H. A. Dassonville, Proc. SPIE 8636, 863610 (2013).

[11] E. Arthurs and J. Kelly, Jr., Bell Syst. Tech. J 44, 725 (1965).

[12] Princeton Instruments, PyLoN 400BR eXcelon.

[13] Given that the raw CCD data are real, the FFT output is symmetric about the midpoint.

[14] R. Loudon, The Quantum Theory of Light (Oxford University Press, New York, 2000).

[15] D. W. Scott, Multivariate Density Estimation: Theory, Practice, and Visualization, Vol. 383 (John Wiley \& Sons, Hoboken, NJ, 2009).

[16] E. Bimbard, R. Boddeda, N. Vitrant, A. Grankin, V. Parigi, J. Stanojevic, A. Ourjoumtsev, and P. Grangier, Phys. Rev. Lett. 112, 033601 (2014). 